\title{
Die Lehre an der Universität Jena als Beitrag zur deutschen Debatte um Lavoisiers Chemie
}

Jan Frercks

\section{Summary}

The German debate about Lavoisier's chemistry started late, was confined to the theory of combustion, mainly focused on two experiments and was closed relatively soon. The perspective from academic teaching allows a better understanding of these features. There was a far-reaching correspondence between the usual practice of academic chemistry teachers and the epistemic criteria taken to be relevant during the debate about Lavoisier's chemistry. A case study based on textbooks written or used at the University of Jena between 1790 and 1800 allows drawing conclusions about why and how academic chemists succeeded in integrating what was seen as the "chemical revolution" without revolutionising their own practice. If the debate had any profound impact on German chemistry at all, it was the consolidation of the rupture between academic teachers and their apothecary colleagues.

Keywords: Antoine-Laurent Lavoisier; history of chemistry; University of Jena; chemical revolution

\section{Zusammenfassung}

Die deutsche Debatte um Lavoisiers Chemie begann spät, war beschränkt auf die Theorie der Verbrennung, war fokussiert auf nur zwei Experimente

\footnotetext{
* Für wertvolle Kommentare zu einer früheren Version des Manuskripts danke ich Thomas Bach, Olaf Breidbach, Peter Hallpap, Michael Markert und Katja Regenspurger. Die Arbeit entstand mit finanzieller Unterstützung der Deutschen Forschungsgemeinschaft im Rahmen des Sonderforschungsbereiches 482 «Ereignis Weimar-Jena. Kultur um 1800».
}

Dr. Jan Frercks, Friedrich-Schiller-Universität Jena, Institut für Geschichte der Medizin, Naturwissenschaft und Technik, Berggasse 7, D-07745 Jena (jan.frercks@uni-jena.de). 
und wurde relativ schnell beendet. Aus Sicht der universitären Lehre ist dies besser zu verstehen. Es gab eine weitreichende Übereinstimmung zwischen der alltäglichen Praxis der Chemieprofessoren und den in der Debatte um Lavoisiers Chemie als relevant erachteten epistemischen Kriterien. Eine Fallstudie zu den an der Universität Jena zwischen 1790 und 1800 benutzten oder verfassten Lehrbüchern macht deutlich, wie akademische Chemiker die «Chemische Revolution» integrieren konnten, ohne ihre eigene Praxis revolutionieren zu müssen. Wenn die Debatte einen strukturellen Effekt auf die Chemie in Deutschland hatte, dann in der Verfestigung der Trennung unter den Chemikern zwischen den akademischen Lehrern und den Apothekern.

\section{Die Lavoisier-Rezeption in Deutschland}

Verspätet, halbherzig, nationalistisch voreingenommen. Diese Schlagworte charakterisieren das gängige Bild der Lavoisier-Rezeption in Deutschland. In der Tat begann die offene Auseinandersetzung erst 1792, zu einem Zeitpunkt also, als in Frankreich die Mehrheit der Chemiker längst von Lavoisiers Chemie überzeugt war ${ }^{1}$. In seinem Traitéélémentaire de chimie von 1789 fasste Antoine-Laurent Lavoisier (1743-1794) seine Forschungen systematisch zusammen, im selben Jahr wurde mit den Annales de chimie ein Sprachrohr für die neue Chemie gegründet, und bereits 1787 war eine ganz im Sinne Lavoisiers reformierte Fachsprache publiziert worden².

Auch im übrigen Europa erfolgte die Akzeptanz parallel oder nur wenig später als in Frankreich, jedenfalls deutlich früher als in Deutschland ${ }^{3}$. Hier gab es zwischen 1792 und 1794 eine intensive, zuweilen hitzige Debatte, die vor allem in Lorenz Florenz Friedrich von Crells (1745-1816) Chemischen Annalen für die Freunde der Naturlehre, Arzneygelahrtheit, Haushaltungskunst und Manufacturen, in Friedrich Albrecht Carl Grens (1760-1798) Journal der Physik und in dem Intelligenzblatt der in Jena erscheinenden Allgemeinen Litteratur-Zeitung ausgetragen wurde. Karl Hufbauer, der diese Debatte minutiös aufgearbeitet hat, sieht in ihrem Funktionieren, insbesondere in ihrer vergleichsweise schnellen Schliessung, ein Indiz dafür, dass in Deutschland eine funktionierende chemical community schon vor 1800 etabliert war ${ }^{4}$. Unabhängig von der jeweiligen beruflichen Einbindung fand

1 Perrin 1990.

2 Guyton de Morveau et al. 1787.

3 Siehe dazu die Beiträge in Bensaude-Vincent/Abbri 1995, in denen eine Studie zum deutschsprachigen Raum fehlt.

4 Hufbauer 1982. 
eine überregionale, durch das junge Medium der Fachzeitschrift ermöglichte Diskussion unter Personen statt, die sich selbst als Chemiker sahen.

Dies trifft zu, ist aber nur die halbe Geschichte. Insbesondere bleibt unklar, warum die offene Debatte so spät begann, warum sie mit der Theorie der Verbrennung auf einen engen Teilbereich von Lavoisiers Chemie beschränkt war und warum in Deutschland viele Chemiker am Phlogiston festhielten. Dies alles wird plausibler, wenn man die Auseinandersetzung aus Sicht der universitären Lehre betrachtet. Dann zeigt sich, dass die Rezeption von Lavoisiers Chemie durchaus schon in den 1780er Jahren begonnen hatte, wobei allerdings kein Anlass für eine offene Debatte bestand, weil die Sauerstofftheorie problemlos in das dominierende Paradigma des «freien Theoretisierens» passte. Dies erklärt auch das Festhalten am Phlogiston. Einige dieser «phlogistischen» Systeme können durchaus als Weiterentwicklungen von Lavoisiers Theorie gesehen werden. Und die Berücksichtigung der beruflichen Praxis eines massgeblichen Teils der Chemiker in Deutschland, nämlich der Chemieprofessoren, erklärt zumindest teilweise die Beschränkung der Debatte auf die Theorie der Verbrennung sowie das Drängen auf eine schnelle Schliessung.

\section{Eine Chemische Revolution in Deutschland?}

Die im wesentlichen mit Lavoisier in Verbindung gebrachten Neuerungen in der Chemie wurden schon zeitgenössisch als «revolutionär» gesehen und bezeichnet $^{5}$. Diese Sicht prägte dann bis vor wenigen Jahrzehnten auch die Historiographie der Chemie. Es war die Chemische Revolution, die Thomas Kuhn Modell für seine Theorie wissenschaftlicher Revolutionen stand ${ }^{6}$. Mittlerweile wird kaum noch unbefangen von der Chemischen Revolution gesprochen. Zunehmend werden die Kontinuitäten zur Chemie vor Lavoisier oder die Unvollständigkeit des Wandels im Vergleich zur Chemie nach Lavoisier betont, so dass der Anteil Lavoisiers an dem Prozess in Frage steht. Vor allem aber ist weniger denn je offensichtlich, worum es in der Chemischen Revolution im Kern ging.

Einigkeit besteht darin, dass es um mehr ging als um die Ersetzung der durch Georg Ernst Stahl (1659-1734) begründeten Phlogistontheorie durch Lavoisiers Sauerstofftheorie. Strittig bleibt aber, worin die fundamentalen Neuerungen bestehen. Genannt werden insgesamt (1.) die Zusammenset-

5 Siehe Guerlac 1976 und Gough 1982.

6 Darauf weist Hoyningen-Huene 1998 hin, der allerdings - anders als hier vertreten - Kuhns Theorie (Kuhn 1962) mit der Geschichte ganz in Übereinstimmung sieht. 
zung der Luft, (2.) das Konzept der Gase als durch Zufuhr von calorique luftförmig gemachter Stoffe, (3.) die Theorie der Verbrennung, (4.) die Theorie der Atmung, (5.) die Theorie der Säurebildung, (6.) eine pragmatische Konzeption chemischer Elemente, (7.) die Ersetzung chemischer Prinzipien durch spezifische Reaktionsmöglichkeiten, (8.) der Vorrang quantitativer, insbesondere gravimetrischer Analysen in geschlossenen Systemen, (9.) die Übernahme physikalischer Denk- und Arbeitsweisen in die Chemie, (10.) neue Instrumente (Kalorimeter, Gasometer), (11.) die Berücksichtigung der Philosophie Etienne Bonnot Condillacs (1714-1780) und (12.) die neue Nomenklatur ${ }^{7}$. Deren Gewichtung und ihr innerer Zusammenhang sind jedoch weiterhin umstritten.

Damit stellt sich die Frage, wie der entsprechende Prozess in Deutschland $\mathrm{zu}$ charakterisieren ist. Immerhin sprach man ja auch hier schon zeitgenössisch von einer «Revolution ${ }^{8}$ und polarisierte zwischen «Phlogistikern» und «Antiphlogistikern». Auch in der Sekundärliteratur sprechen einige Autoren auf Deutschland bezogen von einer «Revolution» 9 .

Wenn im folgenden argumentiert wird, die Vorgänge rechtfertigen nicht die Bezeichnung «Revolution», so nicht deshalb, weil die Vorgänge in Frankreich keine Revolution wären. Eine diesbezügliche Beurteilung ist im Rahmen dieses Artikels weder möglich noch erforderlich. Es ist aber auch nicht etwa deshalb keine Revolution, weil die Vorgänge in Deutschland «nur» Rezeption und Adaption waren. Ein Verdienst von Kuhns Theorie besteht ja darin gezeigt zu haben, dass Paradigmen verinnerlicht werden müssen - von der scientific community insgesamt wie auch von jedem einzelnen Wissenschaftler. Es geht hier um die Anpassung der eigenen Praxis, nicht um das blosse Einsehen der Überlegenheit des Neuen ${ }^{10}$.

Um eine Revolution handelt es sich vielmehr aus den folgenden Gründen nicht. Erstens erscheint im Lichte der neueren Forschungen zu Frankreich die deutsche Debatte seltsam beschränkt. Es ging vor allem um die Theorie der Verbrennung. Die Einengung auf die Frage «Phlogiston oder Sauerstoff ${ }^{11}$ darf aber nicht als selbstverständlich hingenommen werden, sondern

7 Ohne Anspruch auf Vollständigkeit zusammengestellt aus den Beiträgen in Donovan 1988a sowie Levere 1990, Brock 1992, Holmes 1997 und McEvoy 2000.

8 Ebenfalls ohne Anspruch auf Vollständigkeit: Gren, zitiert nach Vopel 1972, 193; Hermbstaedt, zitiert nach Engel 1994, 217; Alexander von Humboldt, zitiert nach Engel 1994, 228; Lichtenberg in Erxleben ${ }^{6} 1794$, xxii und xxiii; Scherer 1795a, vii; Wolff, zitiert nach Hufbauer $1982,107$.

9 Hufbauer 1982, 84 et passim; Schneider 1992, 43; Laupheimer 1992, 9; Seils 1995, 159 und 163.

10 Daher stellen Rezeptions- und Durchsetzungsphänomene für ein Verständnis von Wissenschaft einen ebenso wichtigen Gegenstandsbereich dar wie die (vermeintlichen) Ursprünge und Neuerungen.

11 Dies ist der Titel von Laupheimer 1992. 
stellt selbst ein erklärungsbedürftiges Faktum dar. Dies ist zu berücksichtigen, wenn es im folgenden hauptsächlich um die Theorie der Verbrennung gehen wird. Einige Gründe dafür, warum die übrigen elf Punkte kaum eine Rolle spielten, werden am Schluss vorgebracht.

Zweitens stellt für die Chemie in Deutschland (und für die Physik gleichermassen) die Ersetzung einer Theorie durch eine andere und eines Stoffes (Phlogiston) durch einen anderen (Sauerstoff) überhaupt nichts Revolutionäres dar, sondern passt genau in die übliche Praxis der Erstellung von Systemen aus einem Repertoire an Stoffen, um damit die bekannten Fakten zu erklären. Anhand von Gren, Johann Heinrich Voigt (1751-1823) und Sigismund Friedrich Hermbstaedt (1760-1833) wird im folgenden deutlich gemacht, wie kreativ dies geschah.

Wenn die Debatte um Lavoisiers Chemie einen strukturellen Effekt hatte, dann in der Spaltung zwischen den Apothekern und den Professoren unter den Chemikern ${ }^{12}$. Dies erklärt dann auch, warum es überhaupt zu einer offenen Kontroverse kommen konnte, die aus diesem Grund nachfolgend kurz dargestellt werden soll.

\section{Der Streit um die Reduktion des roten Quecksilberkalks}

Gegenstand der Debatte war die Theorie der Verbrennung. Man war sich insoweit mit Lavoisier einig, dass diese anhand der Verkalkung (heute: Oxidation) der Metalle als einer besonderen Form der Verbrennung studiert werden soll, weil Verkalkungen reversibel sind. Als besonders aussagekräftig galt hierbei der rote Quecksilberkalk (heute: $\mathrm{HgO}$ ), weil sich dieser durch Hitze allein, das heisst ohne Zusatz weiterer Stoffe, reduzieren lässt.

Roter Quecksilberkalk (mercurius praecipitatus per se) wird hergestellt, indem Quecksilber am Boden einer lang ausgezogenen, aber offenen Phiole über Monate ${ }^{13}$ in einem Sandbadofen auf einer Temperatur gehalten wird, bei der es verdampft, aber nicht glüht. Dann setzt sich auf dem Quecksilber ein rotes Pulver ab. Wenn man dieses nun stark erhitzt, wird nach Lavoisier Sauerstoffgas frei, eine Verbindung aus oxygène und calorique, und das Quecksilber wird aus dem Kalk «wiederhergestellt». Nach der Phlogiston-

12 Die Entstehung der Chemie als Hochschulfach und damit des Typus des Chemikers als Professor beschreibt Meinel 1987. Nach Homburg 1998 stellten die technischen Fabrikanten neben den Professoren die zweite Gruppe der Chemiker, die jedoch in dieser Debatte nicht in Erscheinung traten. Lediglich Hermbstaedt war vor und Trommsdorff lange nach der Debatte in der technischen Chemie tätig, siehe Hufbauer 1982.

13 Dies erforderte offensichtlich ein kontinuierlich betriebenes Labor, wie es am ehesten den Apothekern zur Verfügung stand. 
theorie muss man für die Wiederherstellung Phlogiston bereitstellen, das sich mit dem Kalk zu Quecksilber verbindet. Hier aber funktioniert die Reduktion ohne externe Phlogistonquelle (Kohle, Öl o.ä.), was das Phänomen zu einem Prüfstein der Theorie macht. In der Tat waren sich die Beteiligten darüber einig, dass dieses Experiment das experimentum crucis für die gesamte Debatte über die rivalisierenden Theorien darstellte ${ }^{14}$.

In der deutschen Debatte (für die Details siehe Hufbauer 1982) ging es nicht nur um die Interpretation der Resultate von Lavoisiers Experiment, sondern um das Experiment selbst. Die richtige Bereitung des Ausgangsstoffes wurde ebenso in Frage gestellt wie die Durchführung und die Analyse der Produkte - und zwar mittels eigener Experimente. Alle der zentral an der Debatte Beteiligten - Gren, Hermbstaedt, Martin Heinrich Klaproth (1743-1817), Johann Friedrich Westrumb (1751-1819) und Johann Bartholomäus Trommsdorff (1770-1837) - waren ausgebildete Apotheker. Offenbar war eine handwerkliche Ausbildung Voraussetzung dafür, nicht nur mit Argumenten, sondern mit Experimenten in die Diskussion einzugreifen. Die dafür nötigen Fähigkeiten im Umgang mit Stoffen und Geräten fand man am ehesten unter den Apothekern, auch wenn die Mehrheit der Apotheker insgesamt kaum an der Debatte interessiert war ${ }^{15}$.

Diejenigen allerdings, deren berufliche Hauptaufgabe zum Zeitpunkt der Debatte die Lehre der Chemie war, waren es, die entweder von vornherein die neue Chemie vertraten (Hermbstaedt) oder sich bereit fanden, ihre Position anzupassen (Klaproth, Gren). Diese setzten sich schliesslich durch, wobei die Nur-Apotheker sich nicht unbedingt bekehrt zeigten. Sie stiegen einfach aus der theoretischen Debatte aus und widmeten sich dauerhaft (Westrumb) oder eine Zeitlang (Trommsdorff) ausschliesslich der praktischen Chemie.

So aussagekräftig dieser Streit und sein Ausgang für die Binnenstruktur der chemical community in Deutschland ist, so untypisch ist er auch, und zwar, weil er in Form von Experimenten ausgetragen wurde. Experimente waren aber weder für die Apotheker noch für die Professoren eine typische, das heisst ihrem Beruf geschuldete, Form der chemischen Praxis. Für die Apotheker bestand die Chemie aus Arbeiten zur Herstellung von Stoffen. Für die Lehrenden bestand die chemische Praxis zuallererst in der Vermittlung chemischen Wissens. Wenn dabei mit Geräten und Stoffen hantiert wurde,

14 Dass die Reduktion des roten Quecksilberkalks eine gravierende Anomalie der Phlogistontheorie darstellt, ist schon eine wesentliche Konsensleistung, war doch gerade wegen dieses ungewöhnlichen Phänomens lange nicht klar, ob das rote Pulver ein «richtiger» Kalk und Quecksilber überhaupt ein «richtiges» Metall sind, siehe dazu Perrin 1969.

15 Engel 1994, 199. 
dann zum Zweck der Demonstration. Experimente hingegen hatten keinen Ort. Das ist ganz wörtlich zu nehmen. Es ist bezeichnend, dass Hermbstaedt seine ersten gelungenen Versuche in seiner Vorlesung vorführte ${ }^{16}$, und das «öffentliche» Experiment vom 3.4.1793 fand in den Räumen seiner Apotheke statt, nicht etwa in der Akademie $^{17}$.

Der Versuch der Überprüfung von Lavoisiers Experiment mag unter der mangelnden finanziellen und technischen Ausstattung gelitten haben ${ }^{18}$. Wenn Hermbstaedt in der Widmung seiner Übersetzung von Lavoisiers Traité an den preussischen Minister Ewald Friedrich Graf von Hertzberg (1725-1795), gleichzeitig Kurator der Königlichen Akademie der Wissenschaften zu Ber$\operatorname{lin}^{19}$, diesen recht unverblümt um Geld für die zum Nachvollzug von Lavoisiers Experimenten nötigen Geräte bat, weil der dazu nötige «Kostenaufwand [...] die Kräfte eines deutschen Privatmannes bei weitem übersteigt» ${ }^{20}$, wenn Klaproth dieses Anliegen in einer Rede vor der Akademie am 21.6.1792 fast wörtlich wiederholte ${ }^{21}$, wenn Gren nicht selbst experimentierte, weil er sich das Quecksilber als Sperrflüssigkeit des pneumatischen Apparats nicht leisten konnte ${ }^{22}$, und wenn Westrumb geeignete Dichtungen für gasdichte Glasverbindungen erst erfinden musste ${ }^{23}$, so zeigt dies die gegenüber Lavoisier beschränkten Möglichkeiten. Immerhin gehörten diese Chemiker sämtlich zu der von Hufbauer ausgemachten core group der chemical community in Deutschland ${ }^{24}$. Entscheidend ist jedoch, dass diese Form der chemischen Praxis des genauen Messens, ja des Experimentierens überhaupt, keine berufliche Basis hatte.

Die für Chemieprofessoren angemessene Form der chemischen Praxis war die des Vermittelns, Erklärens und Vorführens. Das heisst nicht, dass nicht experimentiert wurde, aber dieses stand in enger Beziehung zur Lehre. Dies lässt sich gut an Johann Friedrich August Göttlings (1753-1809) Experiment zur vollständigen Verbrennung von Phosphor verdeutlichen (Details dazu im Kapitel «Techniken der Vermittlung: Göttling»). Nicht ohne Grund war es dieses Experiment, nicht Hermbstaedts, das Gren, die zentrale Figur der Debatte, von der Existenz des Sauerstoffs überzeugte. Dieses Experi-

16 Engel 1994, 240.

17 Engel 1994, 241. Zum Verhältnis Apotheke und Öffentlichkeit, siehe Wahrig 2003.

18 Vopel 1972 sieht hierin einen wesentlichen Grund für die zögerliche Akzeptanz von Lavoisiers Chemie.

19 Engel 1994, 191.

20 Lavoisier 1792, Widmung.

21 Engel 1994, 233-235.

22 Seils 1995, 166.

23 Diese sind beschrieben in einem Brief Westrumbs (abgedruckt in Gren 1792) und kritisiert in Hermbstaedt 1792.

24 Hufbauer 1982, 83ff. 
ment stand in technischer und in epistemologischer Hinsicht Vorlesungsexperimenten nahe. Die Debatte um Lavoisiers Chemie ist daher nur zu verstehen, wenn man die chemische Praxis der akademischen Lehre ernst nimmt. Es zeigt sich so, dass Lehre mehr ist als die Vermittlung des in der Forschung gefundenen Wissens.

\section{Fallstudie: Chemielehre an der Universität Jena}

Wenn die je spezifische chemische Praxis massgeblichen Einfluss auf die Rezeption von Lavoisiers Chemie hatte, so ist es konsequent, diese auch für einen bestimmten Ort dieser Praxis zu untersuchen. Die Universität Jena bietet sich dafür aus den folgenden Gründen an.

Der erste Grund betrifft das zentrale Medium der universitären Lehre, das gleichzeitig die wichtigste Quelle darstellt: das Lehrbuch ${ }^{25}$. Für die Gruppe der Lehrenden unter den Chemikern waren Lehrbücher auf mehrfache Weise wichtig. Lehrbücher anderer halfen bei der Konzeption der eigenen Vorlesung. Eigene Lehrbücher dienten zunächst der Vereinfachung der Vorlesung. Weiterhin dienten sie der Qualifikation, denn Berufungskriterium für bezahlte Professuren waren nicht Forschungsergebnisse, sondern die umfassende Kenntnis eines Gebietes, vorzugsweise nachgewiesen durch ein eigenes Lehrbuch. Für die Disziplin insgesamt stellte dieses Muster der Adaption auswärtiger Lehrbücher, der detaillierten Auseinandersetzung mit ihnen in der Vorlesung und ihrer Modifikation im eigenen Lehrbuch ein nicht zu unterschätzendes Moment der Reflexivität dar. Auch wenn die Lehrbücher der Chemie hinsichtlich ihres Aufbaus, der Auswahl des Stoffes und der Art der Darstellung weit weniger standardisiert waren als etwa die der Physik ${ }^{26}$, beruhten auch hier neue Lehrbücher weitgehend auf älteren. Dieses System erlaubte die graduelle Entwicklung eines Faches, mit Schwerpunkten, aber ohne Zentrum, und zunächst unabhängig von Fachzeitschriften.

In Jena wurde nun die Chemie zwischen 1790 und 1800 fast ausschliesslich von Anfängern der akademischen Lehre gelesen: Göttling und Alexan-

25 Die grundlegende Arbeit zu Lehrbüchern der Chemie dieser Zeit in Deutschland ist Haupt 1987, eine sorgfältig recherchierte Zusammenstellung mit treffenden Charakterisierungen der einzelnen Lehrbücher, jedoch ohne eine über das Material hinausgehende These. Auch bei Laupheimer 1992 spielen Lehrbücher eine grosse Rolle, dienen jedoch dazu, die theoretische Position einzelner Personen zu rekonstruieren, ohne die Einbindung der Lehrbücher in die Vorlesung zu berücksichtigen.

26 Siehe Frercks 2004. 
der Nikolaus Scherer (1771-1824) ${ }^{27}$. Göttling, zunächst Provisor in Heinrich Sebastian Bucholz' (1734-1798) Apotheke in Weimar, wurde auf Betreiben Johann Wolfgang von Goethes (1749-1832) auf Staatskosten zum Studium nach Göttingen und zur Weiterbildung nach Holland und England geschickt und anschliessend im Eilverfahren promoviert, um ab 1789 als bezahlter! - Extraordinarius in der Philosophischen Fakultät Vorlesungen in Chemie und Technologie anzubieten ${ }^{28}$. Scherer, der bei Göttling studierte, begann seine Vorlesungen 1794 und war ab diesem Jahr als Sekretär der Naturforschenden Gesellschaft zu Jena unmittelbar in die Naturforschung in Jena eingebunden, bis er 1797 als Hofchemiker nach Weimar geholt wurde ${ }^{29}$. Bei beiden findet sich das beschriebene Muster des Umgangs mit Lehrbüchern. Auf diese Weise sind in dem Zeitraum in Jena auch die weitverbreiteten Lehrbücher Karl Gottfried Hagens (1749-1829), Hermbstaedts und Grens präsent, so dass die Fallstudie durchaus exemplarisch für die Chemie in Deutschland steht. Insbesondere decken diese Lehrbücher das Spektrum der Positionen zur französischen Chemie ab.

Der zweite Grund ist, dass beide, Göttling und Scherer, an der Auseinandersetzung um Lavoisiers Chemie aktiv beteiligt waren, indem sie die rivalisierenden Theorieentwürfe weiterentwickelten. Sie taten dies nicht in erster Linie in Zeitschriften, sondern in eigenen Büchern. Folgen wir also Göttling und Scherer anhand der von ihnen benutzten und geschriebenen Lehrbücher.

\section{Chemie als Arbeit: Hagen}

Als Göttling im Wintersemester 1789/90 zum ersten Mal die Experimentalchemie las, wählte er als Lehrbuch Hagens Grundri $\beta$ der Experimentalchemie und behielt dieses bis zum Wintersemester 1795/96 bei ${ }^{30}$. Hagen selbst hatte seinen Grundriß für seine Vorlesungen in Königsberg als Lehrbuch der praktischen Chemie in Ergänzung zu den gängigen Lehrbüchern, die von ihm als $\mathrm{zu}$ «theoretisch» ${ }^{31}$ angesehen wurden, verfasst. Der zentrale Gegenstand

27 Der für die Chemie zuständige Ordinarius Ernst Anton Nicolai (1722-1802) las nach 1790 die Chemie gar nicht mehr und der ebenfalls an der Medizinischen Fakultät angesiedelte August Johann Carl Batsch (1761-1802) nur noch sporadisch. Für eine Übersicht über die Vorlesungen im Bereich der Naturwissenschaften siehe Bach/Breidbach 2001 und für die Vorlesungen an der Universität Jena insgesamt Neuper 2003.

28 Zur Biographie Göttlings siehe Schmitson 1811, Schneider 1955 und Stolz 1991a, zum Werk Aigner 1985 und Laupheimer 1992. Als Geburtsjahr Göttlings wird teilweise 1755 genannt.

29 Zur Biographie Scherers siehe Müller 1965.

30 Universitätsarchiv der Friedrich-Schiller-Universität Jena, Akten M 190 bis M 213.

31 Hagen ${ }^{2} 1790$, vii. 
der Auseinandersetzung um Lavoisiers Chemie, die Verkalkung, wird bei Hagen folgendermassen definier ${ }^{32}$ :

Durch Verkalken oder Calciniren (Calcinatio) versteht man in weitläufigerem Sinne diejenige Veränderung eines festen Körpers, wodurch er seinen vorigen Zusammenhang verliehrt, und zerreiblicher wird, indem die Bestandteile, die den Zusammenhang vermittelten, davon entfernt werden. Sonsten wird hierdurch besonders die Veränderung der Metalle verstanden, wodurch sie mit Verlust ihres metallischen Glanzes in einen lockern und pulverichten Zustand versetzt werden.

Verkalkung ist somit zunächst ein praktisches Verfahren, unabhängig von einer theoretischen Interpretation. Dies gilt auch für den Umkehrprozess ${ }^{33}$.

Durch die Wiederherstellung (Reductio), die man im Großen das Anfrischen nennt, giebt man metallischen Kalken alle Eigenschaften eines Metalls, als Zusammenhang, Glanz, Farbe, Dehnbarkeit u.d., wieder zurück.

Erklärt wird die Verkalkung dann nach der Phlogistontheorie. Ursache für die genannten Eigenschaften der Metalle ist demnach das in ihnen enthaltene Phlogiston, das die Metalle bei der Verkalkung verlässt.

Hagen erwähnt durchaus einige Experimente Lavoisiers, im Vordergrund stehen bei ihm aber die verschiedenen Arbeiten. Die thermische Zersetzung von rotem Quecksilberkalk in Quecksilber und «dephlogistisirte» Luft wird nur kurz erwähnt ${ }^{34}$. Quecksilber gewinnt man bei Hagen aus Zinnober und dephlogistisierte Luft aus Braunstein. Es geht um günstige Verfahren zur Herstellung von Stoffen, nicht um theoretisch einfache, aber teure Verfahren zur Darstellung von Stoffen.

Das Buch besteht aus einer Folge von chemischen Arbeiten, die mehr oder weniger detailliert in Form von Anleitungen dargestellt und ohne erkennbares Kriterium angeordnet sind. Die Eigenschaften der Stoffe, Details der Geräte, die chemische Theorie und chemische Experimente kommen durchaus vor, aber in Form von «Bemerkungen» zu bestimmten Arbeiten. Dies entspricht Hagens Auffassung, dass die Chemie sowohl in der Forschung als auch in der Lehre von konkreten Verfahren ausgehen soll.

Die Ausrichtung nicht nur des Lehrbuchs, sondern auch der Vorlesung an chemischen Arbeiten bringt allerdings Probleme mit sich. Diese eignen sich nur bedingt als Demonstrationsversuche. Zum Beispiel benötigen viele Arbeiten mehr Zeit als die Vorlesungsstunde, so dass Hagen von den Studenten erwartet, mehrmals am Tag nach dem Fortgang der Prozesse zu schauen. «Bey andern schiebe ich öfters schon fertige Präparate unter, um desto geschwinder zum Zwecke zu kommen.» ${ }^{35}$

32 Hagen ${ }^{2} 1790,264$.

33 Hagen ${ }^{2} 1790,275$.

34 Hagen ${ }^{2} 1790,276$.

35 Hagen $21790, x$. 
Spätestens 1794 genügte Göttling Hagens Grundriß nicht mehr für seine Vorlesung. Er hatte inzwischen nicht nur eine andere Auffassung zur Theorie der Verbrennung, sondern zum Verhältnis von Experiment und Theorie und zur Didaktik der Chemie überhaupt. Dies wird im Abschnitt «Techniken der Vermittlung: Göttling» erläutert.

\section{Hybridtheorien: Gren}

Scherer las im Sommersemester 1796 und im darauffolgenden Wintersemester nach Grens Grundriß der Chemie. Warum verwendete er dann zuvor nicht Grens Handbuch der gesamten Chemie, sondern Hermbstaedts Systematischen Grundri $\beta$ der Experimentalchemie? Möglich ist, dass Grens Handbuch Scherer als theoretisch nicht auf der Höhe der Zeit erschien. In der Tat findet sich in der Folge von Grens Lehrbüchern eine bemerkenswerte Entwicklung in bezug auf den Umgang mit Lavoisiers Theorie.

Im ersten Band seines Handbuchs von 1787 definiert Gren Verkalkung einerseits unter «Vorkenntnisse zur chemischen Untersuchung» ganz ähnlich wie Hagen, andererseits findet sich ihre Theorie in einem eigenen Kapitel unter den «einfachern Stoffen». Die grundlegenden Fakten der Verbrennung sind unstrittig. Für die Verbrennung wird Luft benötigt, in «reiner Luft» ${ }^{36}$ erfolgt die Verbrennung besser, in einem geschlossenen Gefäss reduziert sich das Volumen der Luft auf höchstens 3/4, und die Kalke sind genau um so viel schwerer, als die übrige Luft leichter ist. Bestätigt wird dies in einem Experiment, bei dem ein Tischlein mit einem Stück Phosphor in eine flache, mit Wasser oder Quecksilber gefüllte Wanne gestellt wird. Über das Tischlein wird eine Glasglocke gestülpt, und dann der Phosphor mit Hilfe eines Brennglases entzündet. Der Phosphor verbrennt, worauf der Wasserbzw. Quecksilberspiegel in der Glocke steigt. Dies ist keine Arbeit im Sinne Hagens, sondern ein Experiment. Hier wird nicht hergestellt, sondern gezeigt. Die Position im Text nach den Fakten macht es zu einem (virtuellen) Demonstrationsexperiment.

Aus Fakten plus Demonstrationsexperiment folgt nun aber noch nicht eine bestimmte Theorie. Vielmehr stellt Gren die Theorien Carl Wilhelm Scheeles (1742-1786), Lavoisiers, Adair Crawfords (1748-1795) und seine eigene dar, die er als «im Grunde alle nicht so sehr voneinander verschieden» ansieht ${ }^{37}$. Die Ausführlichkeit des Abschnitts zur Verbrennung spricht dafür, dass diese ein Thema war. Andererseits ist von einer krisenhaften Situation 
nichts zu merken, Lavoisiers Theorie ist eine unter mehreren. Nach Lavoisier enthalten brennbare Stoffe kein Phlogiston. In Grens Begriffen ist dafür die «reine Luft» zusammengesetzt aus einer Basis und «dem Stoff des Lichtes, den er auch den Stoff des Feuers, und der Hitze nennt $»^{38}$. Bei der Verbrennung verbindet sich die Basis mit dem brennbaren Körper, und der Licht-Feuer-Hitze-Stoff wird frei.

Hier setzt Grens Kritik an. (A) Die Freisetzung eines Stoffes erklärt nicht, wie Licht und Wärme entstehen. (B) Die Freisetzung von reiner Luft aus Metallkalken ist nicht ausreichend nachgewiesen. In Grens eigener Theorie ist hingegen das Phlogiston aus Lichtstoff und Wärmestoff zusammengesetzt und in brennbaren Körpern gebunden. Bei der Verbrennung wird dieses von der umgebenden Luft angezogen, die dadurch phlogistisiert wird. Wenn sich dabei das Phlogiston zeitweise in seine Bestandteile zerlegt, werden diese als Licht und Wärme wahrnehmbar. Die Gewichtszunahme der Kalke erklärt Gren aus dem Verlust des imponderablen Phlogistons, das somit in der neuen Verbindung das «absolute Gewicht $»^{39}$ der dephlogistisierten Luft verringert. Unter der Glasglocke wird also das Gewicht und das Volumen der Luft vermindert, nicht aber ihre «Materie» ${ }^{40}$.

Die übliche Positionierung der Metalle am Ende eines Chemielehrbuchs erlaubt es Gren, in dem erst 1790 erschienenen vierten Band seines Handbuchs noch einmal ausführlich auf das Thema Verkalkung einzugehen. Die benannten Fakten sind dieselben wie drei Jahre zuvor, auch das demonstrierende Experiment ist im Prinzip dasselbe, nur jetzt dem Thema entsprechend mit Zinn statt mit Phosphor durchgeführt.

Ganz anders ist jedoch der Umgang mit Lavoisier. Zunächst führt Gren weitere Gegenargumente an: (C) Es gibt Verkalkungen ohne Licht und Wärme, Lavoisier erklärt nicht, wo das calorique nach der Trennung von der Basis der Lebensluft bleibt. (D) Die Hitze kann (beim roten Quecksilberkalk) nicht sowohl die Verbindung der Basis der Lebensluft mit dem Quecksilber bewirken als auch die Trennung von diesem. (E) Die meisten Kalke sind nicht sauer, obwohl sie nach Lavoisier die «base oxigyne» ${ }^{41}$ enthalten.

Vor allem aber wird Lavoisiers Experiment zur Reduktion des roten Quecksilberkalks in Frage gestellt. Die Verringerung der Luftmenge in dem Phosphor- oder Zinnexperiment ist für Gren ein Fakt, aber die Freisetzung von dephlogistisierter Luft in dem Experiment mit rotem Quecksilberkalk (noch) nicht. Gren vermutet, dass Lavoisiers Kalk entweder aus der Luft

38 Gren ${ }^{1} 1787-1790, \mathrm{I}, 211$.

39 Gren ${ }^{1} 1787-1790$, I, 219.

40 Gren ${ }^{1} 1787-1790$, I, 220.

41 Gren ${ }^{1} 1787-1790$, IV, 87. 
oder durch die schnelle und einfache Bereitung mit Hilfe von Salpetersäure Wasser aufgenommen hat, und dieses Wasser wird dann beim Glühen des Kalkes als dephlogistisierte Luft freigesetzt ${ }^{42}$. Diesen Fehler aufzuzeigen ermöglicht es ihm dann, das «so künstlich ausgedachte System [Lavoisiers] gerade in seinem Grunde zu erschüttern $»^{43}$.

Zudem trifft das - aus Sicht der Antiphlogistiker - entscheidende Kriterium gerade dieses Experiments, nämlich dass hier keine Phlogistonquelle vorhanden ist, für Gren nicht zu. Wenn Phlogiston aus Lichtstoff und Wärmestoff besteht, ist sein Vorhandensein beim Glühen des Kalks ohnehin gesichert.

Für seine eigene Theorie spezifiziert Gren nun auf Grund von Nachfragen das Konzept des Gewichtsverlustes durch Phlogiston. Da dieses aus Lichtstoff und Wärmestoff besteht, die beide «absolute Leichtigkeit, Repulsionskraft ${ }^{44}$ haben, wirkt das Phlogiston mit einer «der Schwerkraft entgegengesetzten Kraft ${ }^{45}$, so dass es in Verbindungen diese leichter macht. Gren argumentiert, dass es eine unbeweisbare «petitio principii ${ }^{46}$ sei, dass Materie schwer sein müsse. Es sei durchaus möglich, ja in der Tat durch das Phlogiston bewiesen, dass dies nicht für alle Stoffe gelte.

Insgesamt ist die Diskussion im Gegensatz zu 1787 stark polarisiert, das Wort «Antiphlogistiker» ${ }^{47}$ fällt erstmals, und Gren denkt, mit seinem Beitrag «die deutsche Stahlische Lehre vom Phlogiston gerettet zu haben» ${ }^{48}$. In dieser Hinsicht entspricht diese Phase der von Kuhn beschriebenen Krisenphase, in der eine Anomalie (die Gewichtszunahme bei der Verkalkung) als problematisch anerkannt wird, und man versucht, die etablierte Theorie durch Zusatzhypothesen zu retten. Dies gelingt Gren, jedenfalls wenn man bereit ist, die Äquivalenz von Materie und Schwere in Frage zu stellen ${ }^{49}$.

Eigene Ansichten, Argumentation, Diskussion, Polemik, all dies findet sich im Lehrbuch, nicht nur in Zeitschriften. Dies geht weit über das übliche Nachtragen der «neuesten Entdeckungen», wie es häufig in Untertiteln heisst, hinaus. Das Lehrbuch gibt nicht einfach den aktuellen Stand der Diskussion wieder, es ist Teil dieser Diskussion. Insbesondere hat die Re-

42 Dies ist so abwegig nicht, wenn man Lavoisiers hydrogène mit dem Phlogiston identifiziert, wie dies von dem in Deutschland intensiv rezipierten Richard Kirwan (1733-1812) vorgeschlagen worden war, siehe Laupheimer 1992, 65-70.

43 Gren ${ }^{1} 1787-1790$, IV, 189.

44 Gren ${ }^{1} 1787-1790$, IV, 76.

45 Gren ${ }^{1} 1787-1790$, IV, 77.

46 Gren ${ }^{1} 1787-1790$, IV, 77.

47 Gren ${ }^{1} 1787-1790$, IV, 75.

48 Gren ${ }^{1} 1787-1790$, IV, 78.

49 Immerhin erklärt ausschliesslich Grens Theorie die bekannte Tatsache, dass phlogistisierte Luft (heute: gasförmiger Stickstoff) spezifisch leichter ist als dephlogistisierte Luft (heute: gasförmiger Sauerstoff). 
duktion des roten Quecksilberkalks schon hier, also nicht erst in der Debatte in den Zeitschriften zwei Jahre später, seine Bedeutung als experimentum crucis erlangt.

Auch in der zweiten Auflage seines Handbuchs, dessen erster Band 1794 erschien, stellt Gren Lavoisiers System ausführlich dar, aber der Ton ist ein ganz anderer als vier Jahre zuvor. Gren erwähnt Lavoisiers Begriffe «Wasserstoffgas $»^{50}$ und $«$ Sauerstoff $»^{51}$. Er ist zwar von der säuernden Wirkung des letzteren noch nicht ganz überzeugt und hält es immer noch für möglich, dass Wasser die Basis der «reinen Lebensluft» ${ }^{52}$ ist, von «dephlogistisirter Luft» spricht er jedenfalls nicht mehr.

Die Ursache für das Wohlwollen ist, dass er sich durch das Verschwinden der «reinen Lebensluft» beim Verbrennen des Phosphors (siehe Kapitel «Techniken der Vermittlung: Göttling») dazu veranlasst sah, «die vorzüglichsten und unterscheidenden Sätze des Lavoisierschen Systems anzunehmen ${ }^{53}$. Gren sieht jetzt - wie Lavoisier - in der Verbrennung eine Verbindung mit der Basis der Lebensluft, die Theorie der negativen Schwere wird nicht mehr erwähnt.

Gren nimmt jedoch weiterhin einen «Brennstoff» ${ }^{54}$ in den brennbaren Körpern an. Dieser ist jetzt - Jeremias Benjamin Richter (1762-1807) folgend - die Basis des Lichts. Wenn dieser sich mit dem Wärmestoff der Lebensluft verbindet, entsteht Licht, das man in der Flamme sieht. Es kann also keine Rede von einer Inkommensurabilität der Systeme im Sinne Kuhns sein. In Grens Theorie kommen Sauerstoff und Brennstoff vor, und das in einer durchaus konsistenten und den Tatsachen gerecht werdenden Weise. Es handelt sich um eine echte Hybridtheorie ${ }^{55}$.

Die Virtuosität der Erfindung neuer Theorien durch Umdeutung und Rekombination von Grundstoffen in der Abfolge von Grens Theorien ist bemerkenswert. Ebenso auffallend ist die Toleranz in der Beurteilung der Theorien anderer. Zu ein und derselben Theorie heisst es z.B. «diese Theorie $[\ldots]$ hat sehr viel Einnehmendes, und erklärt in mehrern Stücken viel

50 Gren ${ }^{2} 1794-1795$, I, 187.

51 Gren ${ }^{2} 1794-1795$, I, 190.

52 Gren ${ }^{2} 1794-1795$, I, 172.

53 Gren ${ }^{2} 1794-1795$, I, 172.

54 Gren ${ }^{2} 1794-1795$, I, 172.

55 Entsprechend irreführend ist es, Gren (und andere Autoren) der einen oder der anderen Seite zuzuordnen, wie dies Schneider, Laupheimer und Hufbauer tun. Nur in diesem dichotomen Schema (phlogistisch-antiphlogistisch) sind widersprechende Einschätzungen wie «Gren blieb bis zu seinem Tod im Jahre 1798 Phlogistiker» (Laupheimer 1992, 281) und «except for retaining phlogiston as the matter of light in order to explain the radiation accompanying many chemical reactions, he accepted Lavoisier's theory» (Hufbauer 1982, 137) möglich. Sehr differenziert hingegen ist Seils 1995. 
Versuche und Erfahrungen auf eine leichte Weise» und gleich im folgenden Satz «allein bey dem allen überzeugt mich diese Theorie ganz und gar nicht $»^{56}$. Eine andere «faßliche, scharfsinnige, und auf sehr viele Versuche gestützte Theorie» wird im nächsten Atemzug als «unzulänglich zur richtigen Erklärung» bezeichnet ${ }^{57}$. Auch in Grens (von Scherer verwendetem) Grundri $\beta$ der Chemie von 1796 sind Differenzen zwischen den Systemen kein ernstes Problem. Aus Gründen der Einfachheit für die Studenten will Gren nur nach dem «strengen antiphlogistischen Systeme» lehren, fügt aber sofort an, dass es «mit demselben leicht wird, die bis jetzt entwickelten Grundsätze des andern Systems darauf beliebig anzuwenden $»^{58}$.

Das freie Theoretisieren in Verbindung mit weitgehender Toleranz gegenüber den Theorien anderer trifft keineswegs nur auf Gren zu, sondern war weiter verbreitet, wie das Beispiel Voigt zeigt.

\section{Freies Theoretisieren: Voigt}

Voigts Versuch einer neuen Theorie des Feuers, der Verbrennung, der künstlichen Luftarten, des Athmens, der Gährung, der Electricität, der Meteroren, des Lichts und des Magnetismus. Aus Analogien hergeleitet und durch Versuche bestätiget ist ein Buch über das zentrale Thema der Chemie. Dies ist insofern bemerkenswert, da Voigt als Ordinarius für Mathematik neben der Mathematik zwar Physik, aber nie Chemie gelesen hat. Entsprechend ist Voigts Versuch auch kein übliches Lehrbuch. Im Gegenteil, durch die Unabhängigkeit von der universitären Vorlesungsstruktur zeigt sich in ihm die Nähe der Chemie und Physik in Deutschland um 1800 quasi in Reinform. Denn neben den für die Theoriediskussion relevanten Gebieten der Chemie beinhaltet es auch die «chemischen» Teile der Physik, nicht jedoch die in gewöhnlichen Physiklehrbüchern behandelten Gebiete der angewandten Mathematik.

In Voigts Theorie gibt es - analog zu den zwei elektrischen Materien - zwei Brennstoffe. Diese ziehen sich gegenseitig an und setzen bei ihrem heftigen Aufeinandertreffen Lichtstoff frei und erzeugen Wärme, welche bei Voigt jedoch kein eigener Stoff ist. Die beiden Brennstoffe nennt Voigt «männlichen» und «weiblichen» Brennstoff ${ }^{59}$. Ersterer entspricht etwa Stahls 
Phlogiston und verbindet sich mit Wasser zu «entzündbarer Luft» ${ }^{60}$. Zweiterer hingegen verbindet sich mit Wasser zu «dephlogistisirter Luft» ${ }^{61}$.

Voigt lehnt Lavoisiers System nicht aufgrund einzelner Experimente ab, sondern weil dieses mit dem «Oxygen», dem «Hydrogen» und dem «Calorique ${ }^{62}$ auf drei hypothetischen Stoffen beruht, deren Namen zudem zweifelhaft gewählt sind, weil sie in keiner eindeutigen Beziehung zur Erzeugung von Säure, Wasser und Wärme stehen.

Dies ist ein ganz typisches Beispiel für das «freie Theoretisieren», wie es gleichermassen in der Physik und der Chemie üblich war. Der Hintergrund ist die von beiden Fächern geteilte Metaphysik der Grundstoffe. Spätestens ab 1790 gab es in der Physik für jedes Phänomen (Elektrizität, Magnetismus, Wärme, Licht) einen oder mehrere solcher Grundstoffe. Ungeachtet dessen, ob sie für ponderabel gehalten wurden oder nicht, handelte es sich um chemische Stoffe, die je nach Wahlverwandtschaft mit anderen chemischen Stoffen reagierten. Auch in der Physik ging es somit um qualitativ zu erfassende Stoffumlagerungen. Zwischen Phlogiston und elektrischer Materie bestand nicht einfach eine Analogie, sie waren Teil desselben Systems ${ }^{63}$. Das freie Theoretisieren bestand dann in der Konstruktion von speziellen Systemen dieser Stoffe, die in Form einer Kombinatorik die bekannten Phänomene erklären sollten. Dies führte zu einer grossen Zahl ideosynkratischer Systeme, die sich notwendigerweise widersprachen. Bemerkenswerterweise wurde dies jedoch in der Regel einfach hingenommen. Die Frage etwa, ob es eine oder zwei elektrische Materien gibt, blieb über Jahrzehnte ungeklärt. Die Forcierung der Entscheidung über das Phlogiston ist demgegenüber eine Ausnahme.

Die Frühphase der Rezeption Lavoisiers passt genau in dieses Schema. Dessen Theorie der Verbrennung war zunächst nur ein weiteres dieser Systeme, das man zur Kenntnis nahm und auch kritisieren konnte, aber eben innerhalb des herrschenden Paradigmas des freien Theoretisierens. Dies erklärt den späten Beginn der Zuspitzung der Auseinandersetzung. Dass es dennoch zu einer offenen Kontroverse kam, ist im wesentlichen Hermbstaedt zu verdanken.

60 Voigt 1793, 7.

61 Voigt $1793,10$.

62 Voigt 1793, 115.

63 Dass Chemie und der nicht-mathematische Teil der Physik dennoch nicht völlig verschmolzen, ist auf die Trennung beider Fächer an den Universitäten zurückzuführen (Stichweh 1984; Seils 1997; Lind 2000; Frercks 2004). 
Das erste «antiphlogistische» Lehrbuch war Hermbstaedts Systematischer Grundriß der allgemeinen Experimentalchemie von 1791, das Scherer 1794 bis 1796 für seine Vorlesungen verwendete. Hermbstaedt hatte mit seinem Projekt der Übersetzung von Lavoisiers Traité die offene Debatte über die neue Chemie im allgemeinen und die Reduktion des roten Quecksilberkalks im besonderen ausgelöst, indem er Westrumb, Klaproth und Johann Christian Wiegleb (1731-1800) um Kommentare gebeten hatte ${ }^{64}$. Nach Michael Enge ${ }^{65}$ nutzte Hermbstaedt Lavoisiers Chemie ganz gezielt, indem er eine Debatte anstiess, die ihm eine Rolle im Zentrum der chemical community verschaffte. In seinem Grundri $\beta$ war er vorsichtiger und lehrte «beyde Systeme» ${ }^{66}$, weil «mehrere der grössten Chemiker Deutschlands noch zu sehr gegen [Lavoisiers] Lehre eingenommen» waren $^{67}$.Zwischen den Zeilen wird jedoch die Bevorzugung des «neuen Systems» ${ }^{68}$ deutlich.

In der zweiten Auflage von 1800 präsentiert Hermbstaedt jedoch eine «neue eigne Hypothese» ${ }^{69}$. Es geht um das in Lavoisiers Theorie nicht ausreichend geklärte Entstehen von Licht bei Verbrennungen. Hermbstaedt unterscheidet zunächst das Phänomen Licht von dessen materieller Ursache, die er «lichtzeugenden Stoff $»^{70}$ nennt. Hermbstaedt ist - wie die Mehrheit der Physiker und Chemiker in Deutschland - davon überzeugt, dass der lichtzeugende Stoff und der «wärmezeugende Stoff $»^{71}$ nicht identisch sind. In Hermbstaedts Hypothese ist der lichtzeugende Stoff Bestandteil brennbarer Körper. Bei der Verbrennung verbindet er sich mit dem wärmezeugenden Stoff des «säurezeugenden Gases» ${ }^{72}$ zu Feuer.

Im Endeffekt ist dies - bis auf die anderen Bezeichnungen - die RichterGren-Theorie. Laupheimer sieht hierin einen «Rückschritt in das phlogistische Zeitalter» ${ }^{73}$. Die Anordnung der Theorien in Hermbstaedts Lehrbuch - erst Grens und Richters Theorie, dann Lavoisiers, schliesslich seine eigene - deutet jedoch darauf hin, dass Hermbstaedt selbst seine Hypothese eher als Weiterentwicklung von Lavoisiers Theorie sah. In der Tat lässt sich die Theorieproduktion in Deutschland auch anders als eine mehr oder

64 Laupheimer 1992, 58-63.

65 Engel 1994, 191.

66 Hermbstaedt ${ }^{1} 1791$, I, vii.

67 Hermbstaedt ${ }^{1} 1791$, I, 98.

68 Hermbstaedt ${ }^{1} 1791$, I, Vorrede.

69 Hermbstaedt ${ }^{2} 1800-1805$, I, 199.

70 Hermbstaedt ${ }^{2} 1800-1805$, I, 199.

71 Hermbstaedt ${ }^{2} 1800-1805$, I, 200.

72 Hermbstaedt ${ }^{2} 1800-1805$, I, 198.

73 Laupheimer 1992, 336. 
weniger weitgehende Annäherung an Lavoisier interpretieren. Man kann Grens Theorie im Sinne Kuhns als eine Modifikation der Phlogistontheorie ansehen, bei der durch die Erklärung einer Anomalie (Gewichtszunahme bei der Verkalkung) die Theorie insgesamt gerettet werden soll. Man kann aber ebensogut - und ganz analog - Hermbstaedts Theorie als Modifikation der Sauerstofftheorie ansehen, bei der durch die Erklärung einer Anomalie (Lichtentwicklung bei der Verbrennung) die Theorie insgesamt gerettet werden soll. Dass beide Theorien - Grens und Hermbstaedts - in der Sache identisch sind, zeigt nur um so deutlicher, dass von einer Polarität zweier dichotomer oder gar inkommensurabler Systeme keine Rede sein kann.

Dass Licht eines der zentralen Probleme der Chemie wurde, zeigt, dass die Annäherung zwischen Chemie und Physik auch von seiten der Chemie erfolgte. Die Themen Licht und Wärme nehmen in der zweiten Auflage von Hermbstaedts Grundri $\beta$ viel breiteren Raum ein als in der ersten, und es gibt sogar ein Kapitel zur Elektrizität einschliesslich ihrer Geräte. Das Gemeinsame zwischen Physik und Chemie bestand nicht in ihrer Experimentalpraxis und nicht in ihren Lehrveranstaltungen, sondern in ihrer Art zu theoretisieren. Doch dies war nicht unumstritten, wie die Entwicklung bei Scherer zeigt.

\section{Induktion, Repräsentation, Exemplifikation: Scherer}

Als Scherer mit seinen Vorlesungen begann, hatte die Debatte um Lavoisiers Chemie ihren Höhepunkt bereits überschritten. Wie gesehen, wählte Scherer mit Hermbstaedts Systematischem Grundri $\beta$ und dann Grens Grundri $\beta$ der Chemie Lehrbücher, die - was die Theorie der Verbrennung angeht - im Sinne Lavoisiers verfasst waren. Dennoch setzte Scherer sich in seinen eigenen Lehrbüchern, dem Versuch einer populären Chemie von 1795, den Grundzügen der neuern chemischen Theorie ebenfalls von 1795 und den Nachträgen zu den Grundzügen der neuern chemischen Theorie von 1796, mit dieser Theorie auseinander, wobei er die Debatte nutzte, um sich von dem freien Theoretisieren zu lösen. Interessant ist, wie Scherer hier Epistemologie und Didaktik, das heisst die richtige Art, Chemie zu praktizieren, und die Struktur der universitären Lehre, verbindet.

Scherer vertritt in seiner Populären Chemie und in seinen Grundzügen eine dreistufige Epistemologie. Er beginnt mit den Erscheinungen - welche Experimente, einfache Demonstrationsversuche oder lebensweltliche Erfahrungen sein können -, wie z.B. «die glühenden Kohlen in einem Windofen werden durch einen Blasebalg stärker angefacht, als durch den blossen Luft- 
zug» ${ }^{74}$. Daraus gewinnt man die Tatsachen, die Scherer für «ewig» ${ }^{75}$ hält, etwa «das Verbrennen geschieht um desto lebhafter, je reiner die Luft ist, und je mehr ihr Zugang befördert wird» ${ }^{76}$.

Der zweite Schritt ist der Übergang von den Tatsachen zur Theorie. Anders als implizit bei Gren, folgt für Scherer notwendig die richtige Theorie durch «Induktion aus einer grossen Anzahl von Erfahrungen» ${ }^{77}$. Scherer löst das gleichermassen epistemologische wie didaktische Problem, wie man aus einer begrenzten Menge von Erfahrungen auf die notwendigerweise richtige Theorie kommt, durch eine Kaskade von Repräsentationen. Zunächst behauptet er die Theorie der Verbrennung als zentral für die Chemie als Wissenschaft insgesamt ${ }^{78}$, dann nutzt er die Ähnlichkeit zwischen Verbrennung und Verkalkung, weil die Verkalkung zusammen mit der entsprechenden Reduktion ein reversibler Prozess ist, mit dem der für die Verbrennung zentrale Stoff leichter zu finden sein soll. Reduktionen unter Verwendung von Brennstoffen wie Kohle oder Fett sind jedoch zu kompliziert, so dass er nur Reduktionen ohne einen Zusatz eines brennbaren Körpers betrachtet.

Damit ist er bei dem Experiment mit dem roten Quecksilberkalk angelangt $^{79}$. Damit ist aber nicht das Experiment an sich gemeint, wie es in verschiedenen Varianten von Westrumb, Trommsdorff und Hermbstaedt durchgeführt wurde, sondern ein ganz bestimmtes: das Experiment Lavoisiers, dargestellt in dessen Traité, Seiten 33 bis 41. Nicht einmal die Pariser Masseinheiten sind übersetzt. Dieses Experiment steht nun für die Reduktion von rotem Quecksilberkalk an sich, diese für Reduktionen ohne Zusatz von Brennstoffen, diese wiederum für Reduktionen überhaupt, für den Doppelprozess Verkalkung/Reduktion, schliesslich für die Verbrennung und damit für die Chemie insgesamt, denn «auf die Einsicht dieses Phänomens gründet sich die ganze chemische Wissenschaft» ${ }^{80}$.

In der Lehrpraxis zeigt sich Scherers Auffassung weniger daran, dass er neben der Standardvorlesung noch Spezialvorlesungen zum antiphlogistischen System anbot ${ }^{81}$, als vielmehr in der Standardvorlesung selbst. Diese

74 Scherer $1795 \mathrm{a}, 65$.

75 Scherer 1795a, 62.

76 Scherer 1795a, 64.

77 Scherer $1795 \mathrm{a}, 62$.

78 Scherer $1795 \mathrm{a}, 59$ und 62.

79 Man beachte, dass aus Sicht der Phlogistontheorie gerade die Reduktion mit brennbaren Körpern den einfacheren Fall darstellt, die Zuspitzung der Auseinandersetzung auf den roten Quecksilberkalk ist damit schon nicht mehr neutral.

80 Scherer 1795a, 59.

81 Es handelt sich um die Vorlesungen Das antiphlogistische System nach seiner Anleitung, Versuche zur Erläuterung des antiphlogistischen Systems besonders, Die Lehre von den Gasarten und Die Literatur und Nomenklatur der neuesten Chemie. 
Vorlesung wurde nämlich aufgeteilt. Scherer las den praktischen Teil montags, dienstags und mittwochs nach Grens Grundri $\beta$ und den theoretischen Teil donnerstags und freitags ${ }^{82}$ nach seinen Grundzügen, die er eigens dafür verfasst hatte ${ }^{83}$. Die wöchentliche Abfolge von Tatsachen (nach Gren) und Theorie (nach Scherer) entspricht genau seiner Epistemologie des induktiven Schliessens. Gleichzeitig erlaubt die Repräsentation die Überzeugung der Studenten in der Vorlesung. Anders als die Vielzahl der Arbeiten bei Hagen, die die Grenzen der Vorlesung sprengten, konnte hier die grundlegende Theorie der Chemie in einem einzigen Experiment exemplifiziert werden, entweder in Form eines real vorgeführten Experiments oder virtuell im Verweis auf Lavoisiers Experiment. Dieses wird zu einem Exemplar, indem es als ein Einzelnes auf etwas Allgemeines verweist.

Auch wenn Scherer wie Gren oder Voigt mit den Erfahrungen beginnt und mit Theorie endet, geht Scherers Konzeption doch in eine andere Richtung. Noch in seinen Grundzügen hatte Scherer einen Lichtstoff angenommen, von dem noch nicht geklärt sei, ob er Teil der brennbaren Körper oder des Sauerstoffgases sei. Dies liefe auf die Richter-Gren-Theorie heraus, doch schon in den Nachträgen vollzieht Scherer eine radikale Wende. Nicht nur hält er jetzt die Frage nach der Identität von Licht- und Wärmestoff für noch nicht entschieden, er verzichtet auf diese Stoffe überhaupt. Da sie nicht materiell seien, könne man sie nicht rein darstellen, sie blieben damit immer hypothetisch. Die Phänomene des Lichts und der Wärme resultierten aus spezifischen Zusammenwirkungen der materiellen Körper. Diese Zusammenwirkungen seien Gegenstand der Physik. Wenn man sie noch nicht kenne, brauche man nicht als Ersatz Stoffe als Ursachen zu erfinden, ebensowenig wie man für das Phänomen des Schalls einen «Schallstoff» ${ }^{84}$ annehme. Chemie solle sich auf das Hantieren mit materiellen Stoffen beschränken. Wenngleich zunächst ohne Resonanz, findet sich hier ein Gegenentwurf zu der Verschmelzung von Chemie und Physik. Theorie soll etwas grundlegend anderes sein als das freie Theoretisieren in Form einer Kombinatorik hypothetischer Elemente ${ }^{85}$.

Auch bei Göttling finden sich Didaktik und Epistemologie in enger Verbindung, es kommt jedoch ein Drittes hinzu: experimentelle Forschung.

82 Universitätsarchiv der Friedrich-Schiller-Universität Jena, Akte M 204.

83 Scherer 1795b, vii.

84 Scherer 1796, 30.

85 Implizit negiert Scherer damit aber auch die These einer Physikalisierung der Chemie durch Lavoisier. Dessen Messungen des calorique wären nach diesem Verständnis keine Chemie. 


\section{Techniken der Vermittlung: Göttling}

Zunächst brachte Göttling seine Standardvorlesung auf den theoretisch neuesten Stand, indem er spätestens ab dem Sommersemester 1794 die in Hagens Lehrbuch fehlende theoretische Struktur durch «eine allgemeine Uebersicht der gesamten Chemie» ${ }^{86}$, deren gedruckte Version er - im Preis der Vorlesung von «2 Louis d'or in Golde» inbegriffen - seinen Studenten auslegte $^{87}$. Göttling fügte aber nicht nur seine Theorie in die Vorlesung ein, sondern auch seine Experimente. Er hatte diese in seinem Beytrag zur Berichtigung der antiphlogistischen Chemie auf Versuche gegründet zusammengefasst. Dies ist kein Lehrbuch, wurde aber in der Lehre verwendet ${ }^{88}$.

Doch der Einfluss ging auch in die andere Richtung. Das didaktische Prinzip, aus dem Anschaulichen unmittelbar Schlüsse zu ziehen, leitet auch Göttlings Forschung. Die Genese des markantesten Teils von Göttlings Theorie zeigt diese enge Verbindung deutlich. Göttling hatte gefunden, dass Phosphor in reiner Luft zu Phosphorsäure verbrennt. Bei geringer Hitze passiert hier nichts, wohingegen in atmosphärischer Luft oder in Stickluft der Phosphor ohne Wärmeentwicklung leuchtet. Daraus folgert Göttling die theoretische Aussage, dass der von Lavoisier als elementar angesehene Stickstoff in Wahrheit aus Sauerstoff und Lichtstoff zusammengesetzt ist.

Göttling argumentiert wie folgt: Da Licht und Wärme allein auftreten können, muss es einen eigenständigen Lichtstoff geben. Da dieser nicht Bestandteil der Sauerstoffluft ist, muss er Bestandteil des Phosphors sein. Wenn man beim Leuchten des Phosphors in Stickluft auch Phosphorsäure erhält, muss Stickluft Sauerstoff enthalten. (Göttling stimmt mit Lavoisier darin überein, dass Sauerstoffluft - bei Göttling «Feuerstoffluft» - aus Sauerstoff und Wärmestoff - bei Göttling «Feuerstoff» - besteht.) Lichtstoff und Feuerstoff verbinden sich bei der Verbrennung zu Feuer. Da beim Leuchten keine Wärme frei wird, enthält Stickluft aber keinen Wärmestoff. Luftförmig wird der Sauerstoff dagegen durch Lichtstoff, der ebenso wie der Lichtstoff des Phosphors frei wird, was die besondere Helligkeit erklärt. Stickstoffluft besteht also aus Sauerstoff und Lichtstoff.

Hier haben wir ein weiteres System der Imponderabilien, das allerdings kaum Anerkennung fand. Wesentlich einflussreicher war ein anderes Experiment, das zudem noch deutlicher die enge Verbindung epistemischer und didaktischer Kriterien zeigt. Göttlings Experiment zur Verbrennung von Phosphor überzeugte Gren davon, dass Verbrennung im wesentlichen eine

86 Göttling o.J., 7.

87 Universitätsarchiv der Friedrich-Schiller-Universität Jena, Akte M 200.

88 Universitätsarchiv der Friedrich-Schiller-Universität Jena, Akte M 200. 
Verbindung des verbrennenden Stoffes mit dem Sauerstoff der Luft ist ${ }^{89}$. Wie konnte dieses Experiment eine solche Wirkung entfalten?

Die Technik der Vermittlung bestand in diesem Falle zunächst aus einem neuen Gerät ${ }^{90}$. Üblicherweise legte man ein Stück Phosphor in ein Glasgefäss, füllte dieses mit «reiner Luft», schloss das Gefäss und entzündete den Phosphor, zum Beispiel mit einem Brennglas. Dabei zersprang allerdings das Glas meist. Göttlings Apparat bestand demgegenüber aus einem Messingkolben ( «A» in Abb. 1), der in einem Wasserbad («C») gekühlt werden konnte. Nach dem Verbrennen des Phosphors in reiner Luft öffnete man den Kolben und liess Wasser darin aufsteigen. Bei Göttlings ersten Versuchen füllte das Wasser den Kolben nicht ganz, aber als er die reine Luft aus rotem Quecksilberkalk statt aus dem (billigeren) Braunstein gewann, füllte das Wasser den Kolben nach dem Verbrennen restlos. Dies wurde als klarer Beweis dafür gesehen, dass der Sauerstoff der reinen Luft sich mit dem Phosphor verbunden hatte.

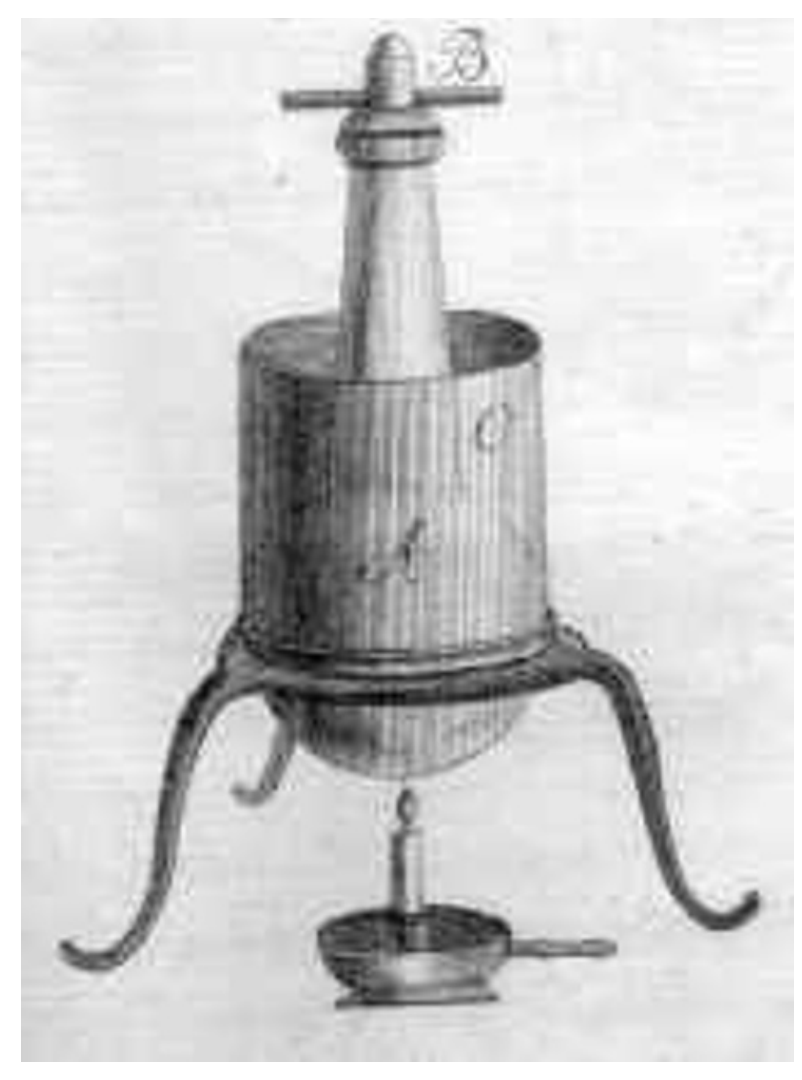

Abb. 1: Die Apparatur für Göttlings Experiment zur Verbrennung von Phosphor (Göttling 1794-1798, I, 9).

89 Scherer 1795b, 50; Göttling 1794-1798, I, Vorbericht; Gren 1793; Ziche/Bornschlegell 2000. 90 Göttling 1794-1798, I, 8-12; Göttling 1794. 
Abb. 2: Die Apparatur für Lavoisiers Experiment zur Verbrennung von Phosphor (Lavoisier 1789, II, Tafel IV).

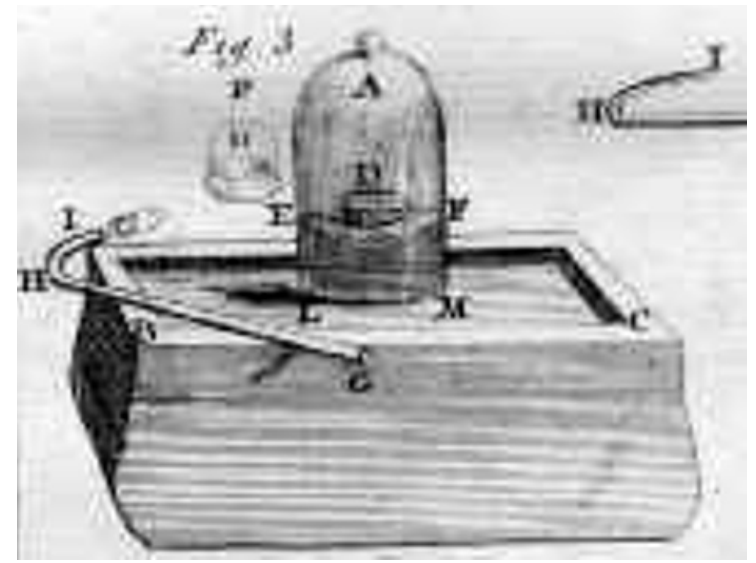

Wenn Gren, wie erwähnt, Göttlings Experiment als Anlass für die Reformulierung seines Systems angibt, ist es zunächst überraschend, dass er in seinem Handbuch nicht dieses, sondern Lavoisiers Experiment beschreibt ${ }^{91}$. Dass man sich bei jedem Zugeständnis an Lavoisiers Chemie nachträglich auf dessen Experimente bezog, ist an sich nichts Ungewöhnliches. Es stellt sich allerdings die Frage, warum dieses Experiment nicht schon früher für Gren überzeugend war.

In Lavoisiers Experiment (Abb. 2) befand sich der Phosphor auf einer kleinen Schale, die auf dem Quecksilber schwamm. Es konnte hier nicht so viel Phosphor verbrannt werden, dass das Sauerstoffgas restlos aufgebraucht würde, weil dann das Schälchen an die Glaskuppel gedrückt würde ${ }^{92}$. Stattdessen wog Lavoisier den Phosphor und die entstehende Phosphorsäure, mass den Anstieg des Quecksilbers und stellte so fest, dass die Gewichtszunahme genau dem Verlust an Sauerstoffgas entsprach.

Warum war dieses Experiment nicht überzeugend? Das Problem ist nicht primär eines der Ausstattung mit Geräten, denn man benötigt dafür im wesentlichen nur einen gewöhnlichen pneumatischen Apparat. Lavoisiers Experiment ist jedoch nur dann einleuchtend, wenn man von der Theorie schon überzeugt ist. Die Volumenverringerung ist dann auf den Verbrauch an Sauerstoffgas zurückzuführen, und das restliche Gas ist einfach das nicht verbrauchte Sauerstoffgas. Phlogistisch interpretiert ist die Luft noch vollständig da, hat jedoch durch die Phlogistisierung an Schwere und Volumen eingebüsst. Erst wenn überhaupt kein Raum mehr übrigbleibt, ist es schwer zu erklären, wo die phlogistisierte Luft geblieben ist. 
Genau dies zeigt Göttlings Experiment. Nicht das in Deutschland unübliche quantitative Experimentieren an sich stellte das Problem dar, sondern der unterschiedliche theoretische Hintergrund selbst. Lavoisiers Experiment setzt Lavoisiers Theorie voraus. Ist man allerdings einmal überzeugt, ist auch Lavoisiers Experiment überzeugend. Warum war dann aber Göttlings Experiment direkt überzeugend? Göttling benutzte das Gerät als Eudiometer zur Bestimmung der Luftgüte ${ }^{93}$. Der Kolben wurde an unterschiedlichen Orten mit Luft gefüllt (indem man Wasser einfüllte und wieder ausgoss), verschlossen und später im Labor analysiert, indem die Menge des nach der Verbrennung des Phosphors aufsteigenden Wassers gemessen wurde. Dies stellt eine Feldmessung dar, und dafür muss das Gerät und das Verfahren einfach, mobil, standardisiert, zuverlässig und nicht sehr zeitaufwendig sein. Nimmt man nun Göttlings Epistemologie hinzu, bei der aus einfachen Experimenten unmittelbar Schlüsse gezogen werden, so eignet sich dieses Experiment perfekt für Vorführungen in Vorlesungen. In der Tat schreibt Göttling:

Ich wiederholte den Versuch verschiedenemal, und weil er mir äußerst wichtig schien, auch in Gegenwart meiner Zuhörer, wo er immer eben so wie das erste mal zutraf. ${ }^{94}$

Einfache Wiederholbarkeit mit sicherem Ergebnis und unmittelbar einleuchtenden Konsequenzen, dieses vorlesungsoptimierte Set an Kriterien war es, das das Experiment für Gren überzeugend machte. Technische und theoretische Einfachheit sind in das Gerät einschliesslich seiner Handhabung eingebaut und vermitteln zwischen Theoriediskussion, chemischer Alltagspraxis und Demonstration in der Lehre.

Für die Lehre an der Universität Jena sind somit vielfältige Verflechtungen mit der Diskussion um Lavoisiers Chemie deutlich geworden. In Erweiterung des Blicks sollen daraus nun einige Schlussfolgerungen für die deutsche Debatte insgesamt gezogen werden.

\section{Schlussfolgerungen}

Die deutsche Debatte um die französische Chemie lässt sich folgendermassen spezifizieren. (1.) Sie betraf mit der Theorie der Verbrennung nur ein Teilgebiet der Chemie. (2.) Sie setzte spät ein. (3.) Sie war hauptsächlich auf zwei Experimente fokussiert. (4.) Sie wurde schnell beendet. Diese Aspekte lassen sich aus Sicht der universitären Lehre besser verstehen.

93 Göttling 1794.

94 Göttling 1794, 141f. 
In den Detailstudien zu den Lehrbüchern Hagens, Grens, Hermbstaedts, Scherers und Göttlings stand von den eingangs erwähnten zwölf Punkten hauptsächlich die Frage nach der Theorie der Verbrennung im Mittelpunkt. Aus unterschiedlichen Gründen kommen die übrigen elf Punkte in den Lehrbüchern kaum vor.

Zum einen gibt es Aspekte, die im Zuge der Theoriediskussion ohne grosse, eigenständige Debatte mit übernommen wurden. Dies betrifft die Theorie der Gase, die mit einem «expansiven» Wärmestoff für die deutsche Metaphysik kein Problem darstellte. Dies betrifft ebenso die Theorie der Atmung, die als Anwendung des Phlogistonkonzepts behandelt und entsprechend später mittels des Sauerstoffkonzepts erklärt wurde.

Die Reform der chemischen Nomenklatur, auch wenn sie letztlich im Sinne Lavoisiers ausfiel, war ein breites und nicht unbedingt anhand von Lavoisier diskutiertes Problem, das hier nicht genauer erörtert werden $k^{k} n^{95}$. Die Bedeutung der Sprache für das Denken wurde implizit gesehen, wenn zunächst die Theorie selbst mit althergebrachten oder möglichst neutralen Begriffen diskutiert wurde, und erst als die Theorie akzeptiert war, wurden auch Lavoisiers Begriffe übernommen. So schrieb Georg Christoph Lichtenberg (1742-1799) seine Mahnung «Hypothesen gehören dem Verfasser, aber die Sprache gehört der Nation $»^{96}$ erst 1794, als die Diskussion der Inhalte ihren Höhepunkt schon überschritten hatte. Dann übernahm man auch den Begriff «Sauerstoff», blieb jedoch gegenüber Lavoisiers Theorie der Säurebildung durch den Sauerstoff skeptisch.

Andere Aspekte kommen nicht vor, weil sie um 1790 längst etabliert waren. Dies betrifft die Existenz verschiedener Luftarten. Dies betrifft ebenfalls die Abkehr von den Prinzipien (und der Erklärung von Eigenschaften einzelner Stoffe) zugunsten materieller Stoffe (und der Betrachtung von Reaktionen). Wie weit man von dem Konzept der Prinzipien entfernt war, zeigt die häufige Kritik an Lavoisier, mit dem «säurezeugenden» und dem «wasserzeugenden» Stoff gerade derartige Prinzipien wieder eingeführt zu haben. Auch fasste man Elemente schon vor der Lavoisier-Rezeption pragmatisch als mit den zur Zeit verfügbaren Mitteln nicht weiter zerlegbare Stoffe auf.

95 In Ermangelung eines Beitrages in Bensaude-Vincent/Abbri 1995 sei hier vorläufig auf Vopel 1972 verwiesen.

96 In Erxleben ${ }^{6} 1794$, xxxviii. 
Der vielleicht umstrittenste Aspekt von Lavoisiers Chemie ist das Verhältnis zur Physik. Wenn etwa Donovan ${ }^{97}$ die Einführung physikalischer Methoden in die Chemie als zentral für die Chemische Revolution ansieht, ist damit das Ideal und die Praxis von Präzisionsmessungen gemeint. Diese Praxis wurde in Deutschland nicht übernommen, da sie keine soziale und wirtschaftliche Basis hatte. Immer, wenn man in den Lehrbüchern Experimente mit präzisen Mengenangaben beschrieben findet, handelt es sich um Experimente Lavoisiers. Aber wie das Phosphorexperiment gezeigt hat, wurde nicht einmal das Ideal der Präzisionsmessung übernommen. Paradoxerweise hängt dies mit der in Deutschland ganz selbstverständlichen Nähe zwischen Chemie und Physik zusammen, nur war Physik hier eben das Hervorbringen sinnlich wahrnehmbarer Phänomene und die nachherige, rein qualitative Erklärung durch ein Set von Imponderabilien, und gerade nicht Präzisionsmessung.

\section{Integration: Lavoisier als Normalfall}

Dass trotz der vielfältigen Gründe für die Nichtsichtbarkeit der meisten der Aspekte von Lavoisiers Chemie gerade die Theorie der Verbrennung übrigblieb, ist kein Zufall. Hier folgten die akademischen Chemiker der gewohnten Praxis, nämlich dem freien Theoretisieren aufgrund von schriftlich kommunizierten Experimenten anderer. Dies erklärt auch den späten Beginn der offenen Kontroverse, denn Lavoisiers System passte lange in dieses Muster. Auch wenn im Zuge der offenen Debatte nationalistische Töne nicht ausblieben, waren antifranzösische Ressentiments daher nicht der Grund für die «Verspätung $»^{98}$. Nicht die Beschäftigung mit Lavoisiers Theorie begann spät, sondern die Einsicht, dass diese nicht vollständig auf die übliche Weise integrierbar war.

\section{Zuspitzung: Exemplifikation als Prinzip}

Um die Debatte auf wenige Experimente zuspitzen zu können, musste man sich erstens einig sein, dass die Experimente stellvertretend für das Theoriesystem insgesamt stehen, und zweitens mussten die Bedingungen der Experimente unumstritten sein.

Der erste Aspekt war nicht zuletzt durch die Lehrbücher gegeben. Insbesondere bei Gren wird deutlich, dass die Eignung der Reduktion des roten

97 Donovan 1988 b.

98 Als wesentlicher Grund wird dies von Vopel 1972, 245; Hufbauer 1982, 104; Schneider 1989;

Perrin 1990, 275; Stolz 1991b und Brock 1992, 121, genannt. 
Quecksilberkalks als experimentum crucis in den Lehrbüchern schon vorbereitet war. Was nach didaktisch motivierter Eingrenzung des Präsentierten aussieht, nämlich die Konzentration auf ein einzelnes Experiment, wird hier zu einem epistemischen Prinzip der Forschung. Dieses korreliert mit dem Vorlesungsbetrieb. Auswählen, Exemplifizieren, Demonstrieren sind integrale Bestandteile der akademischen Lehrpraxis. Genau diese Momente werden in der Debatte um die französische Chemie als Kriterien der Forschung gegenüber der unbeobachteten Experimentierpraxis der Apotheker durchgesetzt. Göttling führte sein Phosphorexperiment wegen seiner Wichtigkeit den Studenten vor. Von einer epistemologischen Trennung von Forschung und Vermittlung, d.h. auch von der Trennung von massgeblichen Zeugen und blossen Zuhörern, kann hier keine Rede sein. Vielleicht war es die Möglichkeit der Lehrenden, nach Belieben Zeugen rekrutieren zu können, die Trommsdorff veranlasste, gegen die Relevanz von Zeugen generell zu polemisieren ${ }^{99}$.

\section{Schliessung: zurück zur gewohnten Praxis}

Der zweite Aspekt, die einheitliche Experimentierpraxis, war nicht gegeben. Nur so konnte es zu einer ausgedehnten Debatte kommen. Zwar stand mit Crells Chemischen Annalen ein textliches Kommunikationsmedium zur Verfügung, die Schliessung wurde aber erst erreicht, nachdem auch anderes verschickt wurde: Briefe, Personen (junge, hospitierende Studenten oder Apotheker) und der rote Quecksilberkalk selbst. Offensichtlich wurde die textliche Mobilisierung von Experimenten allein als nicht ausreichend betrachtet. Es ging um einheitliche Chemikalien, um Vergleichbarkeit der Geräte, um Wiederholung von Experimenten, um Zeugenschaft, kurz um Standards des Experimentierens.

Ob sich an diesen in der Folge der Übernahme von Lavoisiers Theorie etwas änderte, ist noch genauer zu untersuchen. In der Darstellung jedenfalls zeigen sich geringe Auswirkungen auf die «alltägliche» Chemie. Vitriolnaphta, Milch und Stahl (als Beispiele aus verschiedenen Bereichen der Chemie) finden sich in ihren Eigenschaften und Verfahren um 1800 noch genauso beschrieben wie um $1790^{100}$.

99 Hufbauer 1982, $138 f$.

100 Lediglich bei Göttling 1798-1800 deutet sich mit der Akzeptanz der Elemente Kohlenstoff, Sauerstoff, Wasserstoff und Stickstoff schon eine - wenn auch qualitative - Elementaranalyse an, wenn er etwa Bestandteile der Milch durch Verbrennen im pneumatischen Apparat untersucht. 
Vieles deutet somit darauf hin, dass Chemie in ihrer Breite so weiter betrieben wurde wie zuvor. Alfred Nordmann ${ }^{101}$ sieht hierin sogar das Hauptmotiv für die schnelle Schliessung der Debatte. Lichtenberg konnte als Professor der Physik leicht vor einer zu schnellen Entscheidung warnen. Die Lehrenden der Chemie hingegen wollten nicht auf Dauer konkurrierende Systeme lehren, und die Apotheker wollten zur Tagesordnung zurückkehren. Wenn es Anfang 1790er Jahre grundlegende Veränderungen in der Chemie in Deutschland gab, dann gerade in der Festigung dieser Aufspaltung in die praktische Chemie der Apotheker und die theoretisierende Chemie der Dozenten.

Die Apotheker forschten - wenn überhaupt - zur Verbesserung einzelner Verfahren und Analysemethoden, nicht jedoch zur Erweiterung allgemeinen Wissens ${ }^{102}$. Den Lehrenden boten sich angesichts des experimentierpraktischen Vorsprungs der Apotheker zwei Möglichkeiten. Sie konnten in ihren «Nebenstunden» und mit privat oder durch einzelne Zuweisungen finanzierten Geräten eine kontinuierliche Laborpraxis etablieren, wie dies Göttling, Hermbstaedt und - mit Einschränkungen - Gren taten. Oder sie versuchten dies erst gar nicht, nutzten die Verfügbarkeit empirischer Befunde in den Zeitschriften und betrieben Theorie der Chemie als eine für Professoren adäquate Form der chemischen Forschungspraxis.

Im Umgang mit der französischen Chemie zeigt sich in Deutschland demnach nicht einfach eine funktionierende chemical community etablier ${ }^{103}$, sondern auch eine eigenständige, d.h. insbesondere von Medizin und Apothekenwesen unabhängige, Lehrpraxis. Diese ist so konsolidiert, dass sie mit Lavoisiers Revolution umgehen kann, ohne selbst revolutioniert zu werden. Vielmehr trägt die Praxis der universitären Lehre massgeblich zur Auseinandersetzung mit und zur partiellen Integration von Lavoisiers Chemie bei.

\section{Literatur}

Aigner, Walter, Die Beiträge des Apothekers Johann Friedrich August Göttling (1755-1809) zur Entwicklung der Pharmazie und Sauerstoffchemie (München 1985)

Bach, Thomas/Olaf Breidbach, «Die Lehre im Bereich der «Naturwissenschaften» an der Universität Jena zwischen 1788 und 1807», NTM (2) 9 (2001) 152-176

Bensaude-Vincent, Bernadette/Ferdinando Abbri (eds), Lavoisier in European Context. Negotiating a New Language for Chemistry (Canton, Mass. 1995)

Brock, William H., The Norton History of Chemistry (New York/London 1992)

Dilg, Peter, «Die Apotheke als Forschungsstätte», Berichte zur Wissenschaftsgeschichte 23 (2000) 303-315

101 Nordmann 1986.

102 Dilg 2000.

103 Hufbauer 1982. 
Donovan,Arthur (ed.), The Chemical Revolution. Essays in Reinterpretation (= Osiris [2] 4,1988a)

- "Lavoisier and the Origins of Modern Chemistry", in: Arthur Donovan (ed.), The Chemical Revolution. Essays in Reinterpretation (= Osiris [2] 4, 1988b) 214-231

Engel, Michael, «Antiphlogistiker in Berlin 1789-1793. Versuch der Rekonstruktion einer Scientific community im Theorienstreit», in: Michael Engel (Hrsg.), Von der Phlogistik zur modernen Chemie. Vorträge des Symposiums aus Anlaß des 250. Geburtstages von Martin Heinrich Klaproth, Technische Universität Berlin, 29. November 1993 (Berlin 1994) $168-259$

Erxleben, Johann Christian Polykarp, Anfangsgründe der Naturlehre. Hrsg. von Georg Christoph Lichtenberg (Göttingen ${ }^{6} 1794$ )

Frercks, Jan, «Disziplinbildung und Vorlesungsalltag. Funktionen von Lehrbüchern der Physik um 1800 mit einem Fokus auf die Universität Jena», Berichte zur Wissenschaftsgeschichte 27 (2004) 27-52

Göttling, Johann Friedrich August, «Einige Versuche über die Entzündung des Phosphors in reiner und atmosphärischer Luft», in: Almanach oder Taschenbuch für Scheidekünstler und Apotheker auf das Jahr 1794 (Weimar 1793) 135-144

- Beytrag zur Berichtigung der antiphlogistischen Chemie auf Versuche gegründet, 2 Teile (Weimar 1794-1798)

- Handbuch der theoretischen und praktischen Chemie, 3 Bände (Jena 1798-1800)

- Allgemeine Uebersicht der Chymie, als Einleitung zu Hagens Lehrbuch der Experimentalchymie und einige Erinnerungen die Einrichtung seiner chymischen und technologischen Vorlesungen betreffend (Jena o.J.)

Gough, Jerry B., "Some Early References to Revolutions in Chemistry", Ambix 29 (1982) 106-109

Gren, Friedrich Albrecht Carl, Systematisches Handbuch der gesammten Chemie. Zum Gebrauche seiner Vorlesungen entworfen, 3 Bände (Halle ${ }^{1} 1787-1790,{ }^{21794-1795}$ [4 Bände] und $\left.{ }^{3} 1806-1807\right)$

- «Neue Bestätigung durch Versuche, daß der im Feuer bereitete Quecksilberkalk keine Lebensluft bey seiner Wiederherstellung für sich im Glühen liefert», Journal der Physik 6 (1792) 29-34

- «An das chemische Publikum», Intelligenzblatt der Allgemeinen Litteratur-Zeitung 134 (11.12.1793) 1072

- Grundriß der Chemie. Nach den neuesten Entdeckungen entworfen und zum Gebrauch akademischer Vorlesungen eingerichtet, 2 Bände (Halle ${ }^{1} 1796-1797,{ }^{2} 1800,{ }^{3} 1809,{ }^{4} 1818$ )

Guerlac, Henry, "The Chemical Revolution. A Word from Monsieur Fourcroy”, Ambix 23 (1976) $1-4$

Guyton de Morveau, Louis-Bernard/Antoine-Laurent Lavoisier/Claude Louis Berthollet/ Antoine François de Fourcroy, Méthode de nomenclature chimique, proposée par M.M. de Morveau, Lavoisier, Bertholet, et de Fourcroy. On y a joint un nouveau système de caractères chimiques, adaptés à cette nomenclature, par M.M. Hassenfratz et Adet (Paris 1787)

Hagen, Karl Gottfried, Grundriß der Experimentalchemie. Zum Gebrauch bey dem Vortrage derselben (Königsberg/Leipzig ${ }^{1} 1786,{ }^{2} 1790,{ }^{3} 1796,{ }^{4} 1815$ )

Haupt, Bettina, Deutschsprachige Chemielehrbücher (1775-1850) (Stuttgart 1987)

Hermbstaedt, Sigismund Friedrich, «Einige Anmerkungen über die Entbindung der Lebensluft (gaz oxygène), aus für sich verkalktem Quecksilber, durch bloßes Glühen; nebst Untersuchung derjenigen Einwendungen, welche der Hr. Prof. Gren, und der Hr. Bergcomm. Westrumb diesem Versuche entgegengesetzt haben», Journal der Physik 6 (1792) 422-429

- Systematischer Grundriß der allgemeinen Experimentalchemie, 3 Bände (Berlin ${ }^{1} 1791$, ${ }^{2} 1800-1805$ [4 Bände], ${ }^{3} 1812-1827$ [5 Bände])

Holmes, Frederic L., "What Was the Chemical Revolution About?", Bulletin for the History of Chemistry 20 (1997) 1-9

Homburg, Ernst, "Two Factions, One Profession: The Chemical Profession in German Society 1780-1870", in: David Knight/Helge Kragh (eds), The Making of the Chemist: The Social History of Chemistry in Europe, 1789-1914 (Cambridge 1998) 39-76

Hoyningen-Huene, Paul, "Kuhn and the Chemical Revolution", in: V. M. Abrusci et al. (ed.), Prospettive della logica e della filosofia della scienza. Atti del Covegno Triennale della Società Italiana di Logica e Filosofia delle Science, Roma, 3-5 gennaio 1996 (Pisa 1998) 483-498 
Hufbauer, Karl, The Formation of the German Chemical Community (1720-1795) (Berkeley/ Los Angeles/London 1982)

Kuhn, Thomas S., The Structure of Scientific Revolutions (Chicago 1962)

Laupheimer, Peter, Phlogiston oder Sauerstoff. Die Pharmazeutische Chemie in Deutschland zur Zeit des Übergangs von der Phlogiston- zur Oxidationstheorie (Stuttgart 1992)

Lavoisier, Antoine-Laurent, Traité élémentaire de chimie. Présenté dans un ordre nouveau et d'après les découvertes modernes, 2 Bände (Paris 1789)

- System der antiphlogistischen Chemie, aus dem Französischen übersetzt und mit Anmerkungen und Zusätzen versehen von D. Sigismund Friedrich Hermbstädt, 2 Bände (Berlin/Stettin 1792)

Levere, Trevor H., "Lavoisier: Language, Instruments, and the Chemical Revolution", in:Trevor H. Levere/William R. Shea (eds), Nature, Experiment, and the Sciences. Essays on Galileo and the History of Science in Honour of Stillman Drake (Dordrecht/Boston/London 1990) 207-223

Lind, Gunter, "Chemistry in Physics Textbooks, 1780-1820", in: Anders Lundgren/Bernadette Bensaude-Vincent (eds), Communicating Chemistry. Textbooks and Their Audiences, 17891939 (Canton, Mass. 2000) 119-139

McEvoy, John G., "In Search of the Chemical Revolution. Interpretive Strategies in the History of Chemistry", Foundations of Chemistry 2 (2000) 47-73

Meinel, Christoph, «Zur Sozialgeschichte des chemischen Hochschulfaches im 18. Jahrhundert», Berichte zur Wissenschaftsgeschichte 10 (1987) 147-168

Müller, Rudolf, «Beiträge zur Biographie A. N. Scherers», NTM (1) 2 (1965) 37-55

Neuper, Horst (Hrsg.), Das Vorlesungsangebot an der Universität Jena von 1749 bis 1854 (Weimar 2003)

Nordmann, Alfred, "Comparing Incommensurable Theories. A Textbook Account from 1794", Studies in History and Philosophy of Science A 17 (1986) 231-246

Perrin, Carleton E., "Prelude to Lavoisier's Theory of Calcination. Some Observations on mercurius calcinatus per se", Ambix 16 (1969) 140-151

- "The Chemical Revolution", in: Robert C. Olby et al. (eds), Companion to the History of Modern Science (London/New York 1990) 264-277

Scherer, Alexander Nicolaus, Versuch einer populären Chemie (Mühlhausen 1795a)

- Grundzüge der neuern chemischen Theorie (Jena 1795b)

- Nachträge zu den Grundzügen der neuern chemischen Theorie (Jena 1796)

Schmitson, Anton, Johann Friedrich August Göttling, Kurze Darstellung seines Lebens und seiner Verdienste (Jena 1811)

Schneider, Hans-Georg, "The 'Fatherland of Chemistry'. Early Nationalistic Currents in Late Eighteenth Century German Chemistry", Ambix 36 (1989) 14-21

- Paradigmenwechsel und Generationenkonflikt. Eine Fallstudie zur Struktur wissenschaftlicher Revolutionen: Die Revolution der Chemie des späten 18. Jahrhunderts (Frankfurt a.M. et al. 1992)

Schneider, Wolfgang, «Der Apotheker Göttling», Die pharmazeutische Industrie 17 (1955) 28-31

Seils, Markus, Friedrich Albrecht Carl Gren in seiner Zeit 1760-1798. Spekulant oder Selbstdenker? (Stuttgart 1995)

- «Chemie statt Mathematik. Ein alternatives Programm zur Etablierung der Chemie als eine chemisch-physikalische Naturwissenschaft am Ende des 18. Jahrhunderts», Mitteilungen der Fachgruppe Geschichte der Chemie in der Gesellschaft Deutscher Chemiker 13 (1997) 13-22

Stichweh, Rudolf, Zur Entstehung des modernen Systems wissenschaftlicher Disziplinen. Physik in Deutschland 1740-1890 (Frankfurt a.M. 1984)

Stolz, Rüdiger, «Überzeugter Praktiker und streitbarer Theoretiker. Johann Friedrich August Göttling», Geschichte der Pharmazie 43 (1991a) 55-57

- «Die chemische Revolution des 18. Jh. und ihre Wirkung auf das 19. Jahrhundert», Rostocker Wissenschaftshistorische Manuskripte 20 (1991b) 46-50

Voigt, Johann Heinrich, Versuch einer neuen Theorie des Feuers, der Verbrennung, der künstlichen Luftarten, des Athmens, der Gährung, der Electricität, der Meteroren, des Lichts und des Magnetismus. Aus Analogien hergeleitet und durch Versuche bestätiget (Jena 1793)

Vopel, Hellmut, Die Auseinandersetzung mit dem chemischen System Lavoisiers in Deutschland am Ende des 18. Jahrhunderts (Leipzig 1972) 
Wahrig, Bettina, «Apotheke - Öffentlichkeit - Publikum. Zur Geschichte eines Dreiecksverhältnisses», in: Christoph Friedrich/Wolf-Dieter Müller-Jahncke (Hrsg.), Apotheke und Publikum (Stuttgart 2003) 9-28

Ziche, Paul/Peter Bornschlegell, «Wissenschaftskultur in Briefen. F. A. C. Grens antiphlogistische Bekehrung, galvanische Experimentalprogramme und internationale Wissenschaftsbeziehungen in Briefen an die Jenaer «Naturforschende Gesellschaft»», NTM (2) 8 (2000) 149-169 